\title{
Orientation of multivalent ions near charged planar surfaces
}

\author{
Aleš Razinger ${ }^{1}$, Aleš Iglič ${ }^{1}$ and Veronika Kralj-Iglič ${ }^{1,2}$ \\ ${ }^{1}$ Laboratory of Physics, Faculty of Electrical Engineering, Tržaška 25, SI-1000 Ljubljana, \\ Slovenia \\ ${ }^{2}$ Institute of Biophysics, Medical Faculty, Lipičeva 2, SI-1000 Ljubljana, Slovenia \\ E-mail: ales.iglic@fe.uni-lj.si
}

Received 29 November 2005, in final form 19 January 2006

Published 15 March 2006

Online at stacks.iop.org/JPhysA/39/3275

\begin{abstract}
A solution of large multivalent ions in contact with a planar charged surface is considered theoretically. Using a simple three-state model for the orientation of the multivalent ions in the gradient of an electric field and applying the methods of statistical physics, we show that the internal charge distribution within a single multivalent ion with the charges located at well-separated positions may lead to the orientational ordering of multivalent ions near the charged planar surface.
\end{abstract}

PACS numbers: $41.20 . \mathrm{Cv}, 87.68 .+\mathrm{z}$

\section{Introduction}

Within the Poisson-Boltzmann (PB) theory, ions (including large multivalent ions) are usually described as dimensionless particles bearing a point net charge [1]. However, multivalent ions occupy finite volume [2] and may also have an internal structure with the charges being located at different well-separated positions [3]. Therefore the classical PB description of an electric double layer fails to describe multivalent ions in accordance with experimental observations [4]. Generalization of the PB theory of the electric double layer for the case of multivalent ions can be made by taking into account the excluded volume effect and by considering the internal space charge distribution of a single multivalent ion [5].

Various attempts have been made to incorporate excluded volume (steric) effects into PB theory. Freise [6] introduced the excluded volume effect by a pressure-dependent potential, while Wicke and Eigen [7] used a thermodynamic approach, multiplying the numerical density of ions by a factor containing the number of vacant sites. More recently, the finite size of the ions has been incorporated into PB theory in a more transparent way, based on a lattice statistics model [8-10], a functional approach [11-13] or in a model that takes the surface charge correlation into account, while treating ions and solvent molecules as hard spheres [14]. 


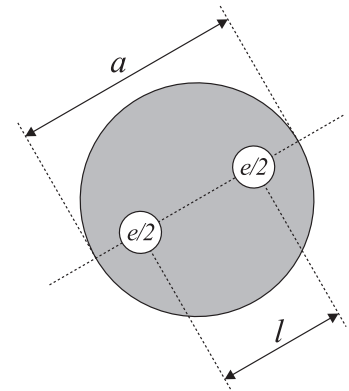

Figure 1. Schematic figure of a large spheroidal multivalent ion with net electric charge $e$ and average radius $a$. In the model, the space charge distribution of the multivalent ion is described by two effective poly-ions of charge $e / 2$ located at different well-separated positions (i.e. at the distance $l \leqslant a$ ). The main axis of the ion coincides with the line connecting the two poly-ions.

In addition to the finite size of the particles, a fluctuation potential has been incorporated [15]. Such approaches lead to better agreement with Monte Carlo (MC) simulations for divalent counterions [16]. Even better agreement of the MC simulations was found for divalent ions when hypernetted chain integral equations were applied [17, 18].

The first modification of PB theory (i.e. considering the excluded volume effect) may lead to better agreement between the calculated and measured density profiles of multivalent ions close to the charged surface $[19,22]$ but it fails to obtain the observed pre-compensation of the charge in a solution of large multivalent ions near the charged plane (overcharging) [4] which can be described by considering the internal distribution of the electric charge in the single multivalent ion [21]. Previously [21] for the sake of simplicity different orientations of multivalent (divalent) ions near the charged surface were taken into account only in the limit of very high temperatures. Therefore the orientational ordering of multivalent ions near the charged plane and its dependence on temperature and other model parameters could not be studied explicitly. In the present work, the orientational ordering of large multivalent ions (figure 1) near the charged plane is studied at finite temperatures. The orientation of the multivalent ions in the gradient of an electric field is described within a three-state model.

\section{Theory}

We consider a system composed of two (very large) parallel uniformly charged planar membranes (plates) of area $A$ which are parallel to the $(x, y)$ plane of an $(x, y, z)$ Cartesian coordinate system. The first charged plate is located at $z=0$, while the second charged plate is located at $z=2 d$. The water solution between the charged plates contains only large multivalent ions, composed in the model of two point effective charges of equal sign $(e / 2)$ separated by a distance $l$ (figure 1). The average electric potential $\Phi$ changes only along the $z$-axis $(\Phi=\Phi(z))$, in the direction perpendicular to the $(x, y)$ (charged) plane. In the electric field gradient $\mathrm{d} E / \mathrm{d} z=-\mathrm{d}^{2} \Phi / \mathrm{d} z^{2}$, the energy of a single multivalent ion depends on the orientation of its main axis (see figure 1 for definition) with respect to the $z$-axis (angle $\vartheta$ ) [21],

$$
U_{e l}=e(\Phi-\widetilde{\Phi})+\frac{e l^{2}}{8} \frac{\mathrm{d}^{2} \Phi}{\mathrm{d} z^{2}} \cos ^{2} \vartheta
$$

where $\widetilde{\Phi}$ is a constant. For the sake of simplicity, in the present work we adopt a simple threestate model for describing the orientation of multivalent ions in the gradient of the electric 
field. The first state that corresponds to the orientation of the main axis of the ion parallel to the $z$-axis $(\vartheta=0)$ has energy (see equation (1))

$$
U_{\leftrightarrow}=e(\Phi-\widetilde{\Phi})+\frac{e l^{2}}{8} \frac{\mathrm{d}^{2} \Phi}{\mathrm{d} z^{2}} .
$$

Two other degenerated states correspond to orientations along the $x$ and $y$ axes, where $\vartheta=\frac{\pi}{2}$ :

$$
U_{\uparrow}=e(\Phi-\widetilde{\Phi}) .
$$

In our model, the space between the charged plates at $z=0$ and $z=2 d$ is divided into thin cells of thickness $\mathrm{d} z$. Within one cell the values of $\Phi$ and $\Phi^{\prime \prime}$ are considered to be constant. We assume that there are $M$ multivalent ions in a single cell; $N$ of them are parallel to the $z$-direction, $N_{1}$ are parallel to the $x$-direction and $\left(M-N-N_{1}\right)$ are parallel to the $y$-direction. The electric energy of a single cell is then

$$
E_{\text {cell }}=N U_{\leftrightarrow}+(M-N) U_{\uparrow},
$$

while the corresponding canonic partition function of the cell is

$$
Q=\sum_{N=0}^{M} \sum_{N_{1}=0}^{M-N}\left(\frac{M !}{N_{1} !\left(M-N-N_{1}\right) ! N !} \mathrm{e}^{-E_{\text {cell }} / \mathrm{k} T}\right) .
$$

From equations (2)-(5) we get

$$
\begin{aligned}
Q & =\exp \left(-\frac{e(\Phi-\widetilde{\Phi}) M}{\mathrm{k} T}\right) \sum_{N=0}^{M}\left(\frac{M !}{N !(M-N) !} \mathrm{e}^{-\beta N}\left(\sum_{N_{1}=0}^{M-N} \frac{(M-N) !}{N_{1} !\left(M-N-N_{1}\right) !}\right)\right) \\
& =\exp \left(-\frac{e(\Phi-\widetilde{\Phi}) M}{\mathrm{k} T}\right) 2^{M} \sum_{N=0}^{M}\left(\frac{M !}{N !(M-N) !}\left(\frac{\mathrm{e}^{-\beta}}{2}\right)^{N}\right),
\end{aligned}
$$

where

$$
\beta=\frac{e l^{2}}{8 \mathrm{k} T} \frac{\mathrm{d}^{2} \Phi}{\mathrm{d} z^{2}}
$$

Using the binomial formula, equation (6) can be rewritten in the form

$$
Q=\exp \left(-\frac{e(\Phi-\widetilde{\Phi}) M}{\mathrm{k} T}\right)\left(2+\mathrm{e}^{-\beta}\right)^{M},
$$

while the average number of ions in the energy state $U_{\leftrightarrow}(\langle N\rangle)$ :

$$
\langle N\rangle=\frac{\exp \left(-\frac{e(\Phi-\widetilde{\Phi}) M}{\mathrm{k} T}\right) 2^{M} \sum_{N=0}^{M}\left(\frac{M !}{N !(M-N) !} N\left(\frac{\mathrm{e}^{-\beta}}{2}\right)^{N}\right)}{\exp \left(-\frac{e(\Phi-\widetilde{\Phi}) M}{\mathrm{k} T}\right) 2^{M} \sum_{N=0}^{M}\left(\frac{M !}{N !(M-N) !}\left(\frac{\mathrm{e}^{-\beta}}{2}\right)^{N}\right)},
$$

can be expressed as

$$
\frac{\langle N\rangle}{M}=\frac{\mathrm{e}^{-\beta}}{\left(2+\mathrm{e}^{-\beta}\right)} .
$$

From the above equation, we also get the average number of ions in the other two states (having the same energy $U_{\uparrow}$ ):

$$
\frac{\langle M-N\rangle}{M}=\frac{2}{\left(2+\mathrm{e}^{-\beta}\right)} .
$$

Figure 2 shows the dependence of $\langle N\rangle / M$ and $\langle M-N\rangle / M$ on the parameter $\beta$. It can be seen in figure 2 that in the high temperature limit when $\beta \rightarrow 0$ the ratio $\langle N\rangle / M$ approaches $1 / 3$ while $\langle M-N\rangle / M$ approaches $2 / 3$ which means that at high temperatures all three 


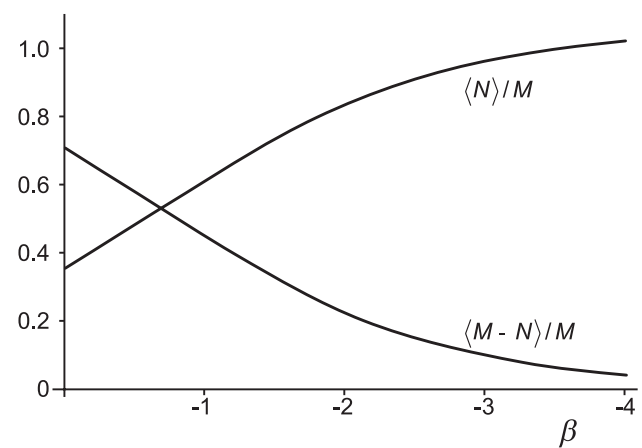

Figure 2. Average relative fractions of multivalent ions (figure 1) orientated along the electric field vector $(\langle N\rangle / M)$ and perpendicular to the electric field vector $(\langle M-N\rangle / M)$ as a function of the parameter $\beta=\frac{e l^{2}}{8 \mathrm{k} T} \frac{\mathrm{d}^{2} \Phi}{\mathrm{d} z^{2}}$.

orientational states are equally occupied. In the low temperature limit (i.e. at large $|\beta|$ ), all ions are oriented along the $z$-axis, i.e. in the direction parallel to the electric field vector.

To derive the total free energy of the single cell $(\Delta F)$, we should also consider the configurational entropy of the ions distributed over the space of the cell, therefore

$$
\mathrm{d} F=-\mathrm{k} T \ln Q+\mathrm{k} T(M \ln M-M)-\mathrm{k} T\left(M_{0} \ln M_{0}-M_{0}\right) .
$$

The third term in equation (12) is the configurational entropy of the reference system [8] in which ions are uniformly distributed so that the corresponding number density of ions $m_{0}=M_{0} / A \mathrm{~d} z$. Taking into account equation (8) we can express the total free energy of the system in global thermodynamic equilibrium as

$$
\begin{aligned}
F=\int \mathrm{d} F= & 2 \int_{0}^{d} e m(\Phi-\widetilde{\Phi}) A \mathrm{~d} z-2 \int_{0}^{d} \mathrm{k} T m \ln \left(2+\mathrm{e}^{-\beta}\right) A \mathrm{~d} z \\
& +2 \mathrm{k} T \int_{0}^{d}\left(m \ln \frac{m}{m_{0}}-\left(m-m_{0}\right)\right) A \mathrm{~d} z,
\end{aligned}
$$

where we introduce the number density of ions $m=M / A \mathrm{~d} z$.

The equilibrium number density of the multivalent ions $(m)$ and the corresponding electric potential are not known in advance. They are obtained using the condition that the free energy of the system is at its minimum at thermodynamic equilibrium of the whole system. The condition for the global equilibrium

$$
\delta F=0,
$$

is subject to the following:

- The local constraint requiring the validity of Gauss's law at any $z$

$$
\varepsilon \varepsilon_{0} \frac{\mathrm{d}^{2} \Phi}{\mathrm{d} z^{2}}+e m=0
$$

- The global constraint requiring a constant number of multivalent ions in the whole system

$$
2 \int_{0}^{d}\left(m-m_{0}\right) A \mathrm{~d} z=0 .
$$


The variational problem can be expressed by Euler-Lagrange equations,

$$
\frac{\partial \mathcal{L}}{\partial E}-\frac{\mathrm{d}}{\mathrm{d} z}\left(\frac{\partial \mathcal{L}}{\partial \frac{\mathrm{d} E}{\mathrm{~d} z}}\right)=0, \quad \frac{\partial \mathcal{L}}{\partial m}=0,
$$

where $\mathcal{L}(m, E, \mathrm{~d} E / \mathrm{d} z)$ is

$$
\begin{aligned}
\mathcal{L}=\frac{1}{2} \varepsilon \varepsilon_{0} E^{2}- & m \mathrm{k} T \ln \left(2+\mathrm{e}^{\frac{e l^{2}}{8 \mathrm{k} T} \frac{\mathrm{d} E}{\mathrm{~d} z}}\right)+\mathrm{k} T\left(m \ln \frac{m}{m_{0}}-\left(m-m_{0}\right)\right) \\
+ & \lambda\left(\varepsilon \varepsilon_{0} \frac{\mathrm{d} E}{\mathrm{~d} z}-e m\right)+\lambda_{m}\left(m-m_{0}\right),
\end{aligned}
$$

where it was taken into account that $E=-\mathrm{d} \Phi / \mathrm{d} z, \lambda$ is the local Lagrange multiplier, and $\lambda_{m}$ is the global Lagrange multiplier. Solving equations (17) gives a second-order nonlinear differential equation for the electric potential $\Phi(z)$,

$$
\Phi^{\prime \prime}=-\frac{e m_{0}}{\varepsilon \varepsilon_{0}}\left(2+\mathrm{e}^{-\frac{e l^{2}}{8 \mathrm{k} T} \Phi^{\prime \prime}}\right) \exp \left(-\frac{e}{\mathrm{k} T} \Phi-\frac{e l^{2}}{8 \mathrm{k} T} \frac{\Phi^{\prime \prime}}{1+2 \mathrm{e}^{\frac{e l^{2}}{8 \mathrm{k} T} \Phi^{\prime \prime}}}-\lambda_{m c}\right),
$$

with the boundary conditions

$$
\begin{aligned}
& \left.\Phi^{\prime}\right|_{z=0}=-\frac{\sigma}{\varepsilon \varepsilon_{0}}, \\
& \left.\Phi^{\prime}\right|_{z=d}=0,
\end{aligned}
$$

where $\Phi^{\prime} \equiv \mathrm{d} \Phi / \mathrm{d} z, \Phi^{\prime \prime} \equiv \mathrm{d}^{2} \Phi / \mathrm{d} z^{2}$ and $\lambda_{m c}$ is a constant. Considering the dimensionless variables,

$$
\xi=\frac{z}{d}, \quad y=\frac{e \Phi}{2 \mathrm{k} T}, \quad \mu=m d^{3},
$$

and the expansion of the exponential functions in equation (19) up to the linear term, we obtain the PB differential equation:

$$
y^{\prime \prime}=-3 \kappa_{\mathrm{D}}^{2} \mathrm{e}^{-2 y} \mathrm{e}^{-\lambda_{m c}},
$$

where

$$
\kappa_{\mathrm{D}}^{2}=\frac{e^{2} d^{2} m_{0}}{2 \varepsilon \varepsilon_{0} \mathrm{k} T} .
$$

The solution of equation (23) can be written as [20]

$$
y=-\frac{1}{2} \ln \left[1+\tan ^{2}\left(\sqrt{3} \kappa_{\mathrm{D}} \mathrm{e}^{-\frac{\lambda m c}{2}}(1-\xi)\right)\right],
$$

where the boundary condition $(21)$ and the choice $y(\xi=1)=0$ were taken into account. The dimensionless number density of ions can then be expressed from Gauss's law (15) as

$$
\mu=3 \mu_{0} \mathrm{e}^{-\lambda_{\mathrm{mc}}}\left[1+\tan ^{2}\left(\sqrt{3} \kappa_{\mathrm{D}} \mathrm{e}^{-\frac{\lambda_{\mathrm{mc}}}{2}}(1-\xi)\right)\right], \quad \mu_{0}=m_{0} d^{3},
$$

while the average fraction of multivalent ions (figure 1) with their main axis parallel to the electric field is derived from equation (10):

$$
\frac{\langle N\rangle}{M}=\frac{\exp \left(\frac{3 \kappa_{\mathrm{D}}^{2} l^{2} \mathrm{e}^{-\lambda \mathrm{mc}}}{4 d^{2}}\left[1+\tan ^{2}\left(\sqrt{3} \kappa_{\mathrm{D}} \mathrm{e}^{-\frac{\lambda \mathrm{mc}}{2}}(1-\xi)\right)\right]\right)}{2+\exp \left(\frac{3 \kappa_{\mathrm{D}}^{2} l^{2} \mathrm{e}^{-\lambda_{\mathrm{mc}}}}{4 d^{2}}\left[1+\tan ^{2}\left(\sqrt{3} \kappa_{\mathrm{D}} \mathrm{e}^{-\frac{\lambda_{\mathrm{mc}}}{2}}(1-\xi)\right)\right]\right)} .
$$

The average fraction of ions perpendicular to the electric field is then

$$
\frac{\langle M-N\rangle}{M}=\frac{2}{2+\exp \left(\frac{3 \kappa_{\mathrm{D}}^{2} l^{2} \mathrm{e}^{-\lambda_{\mathrm{mc}}}}{4 d^{2}}\left[1+\tan ^{2}\left(\sqrt{3} \kappa_{\mathrm{D}} \mathrm{e}^{-\frac{\lambda_{\mathrm{mc}}}{2}}(1-\xi)\right)\right]\right)} .
$$



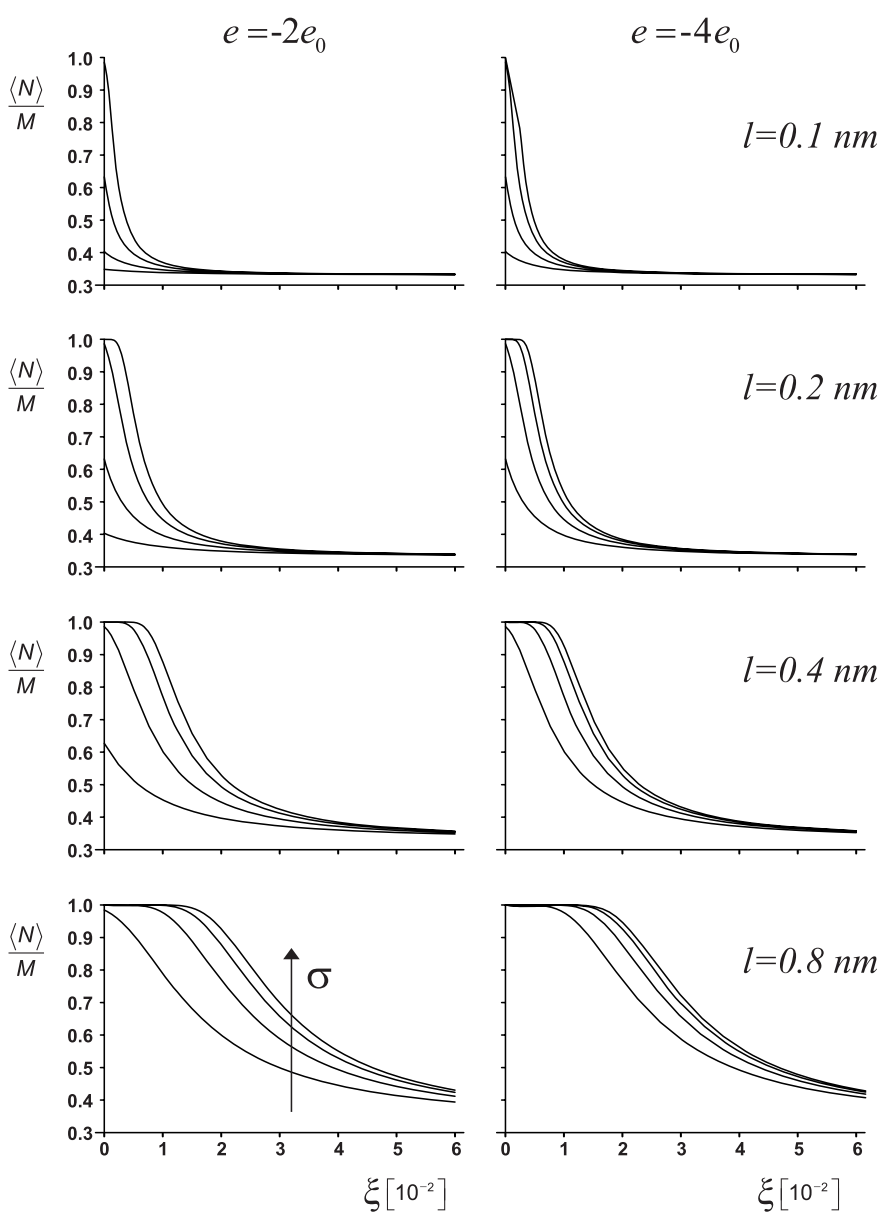

Figure 3. The average fraction of multivalent ions with their main axis parallel to the electric field $\langle N\rangle / M$ as a function of the distance from the charged plane for four different charge densities: $\sigma=0.1 \mathrm{As} \mathrm{m}^{-2}, \sigma=0.2 \mathrm{As} \mathrm{m}^{-2}, \sigma=0.4 \mathrm{As} \mathrm{m}^{-2}$ and $\sigma=0.8 \mathrm{As} \mathrm{m}^{-2}$. Other parameters of the system are $d=10^{-8} \mathrm{~m}, \varepsilon=78, m_{0}=\sigma /|e| d, T=300 \mathrm{~K}$.

\section{Results and discussion}

The parameter $\lambda_{\mathrm{mc}}$ that appears in equations (25)-(28) is numerically calculated from the dimensionless form of the boundary condition (20) for the specific properties of the system determined by the following parameters: surface charge density of both charged plates at $z=0$ and $z=2 d(\sigma)$, distance between the two charged plates $(2 d)$, distance between the effective charges of the single ion $(l)$ (figure 1), dielectric constant of the solution $(\varepsilon)$, temperature of the system $(T)$ and concentration of the solution of multivalent ions $\left(m_{0} / \mathrm{N}_{\mathrm{A}}\right)$, where $\mathrm{N}_{\mathrm{A}}$ is the Avogadro constant.

Figure 3 shows the average fraction of ions with their main axis parallel to the electric field $\langle N\rangle / M$ as a function of their dimensionless distance from the charged plane $(\xi=z / d)$ for four different values of the distance between the effective charges of the ion $l$ (figure 1), two values of the net charge of the single ions $(e)$, and four different values of the surface charge density of the charged plates $(\sigma)$. It can be seen in figure 3 that for $l$ as small as 
$0.1 \mathrm{~nm}$ there is practically no preferred orientational state, even at relatively high surface charge $\sigma$, similarly as in the case of the high temperature limit (figure 2).

With increasing distance $l$ and the absolute value of the net electric charge $e$ of the single multivalent ion, the average fraction of multivalent ions with their main axis oriented in the direction of electric field increases, but only in the close vicinity of the charged surface (figure 3).

In the present paper, the excluded that volume effect $[7-9,11]$ is not taken into account (for example, in equation (12) including configurational entropy) so that the calculated number density of ions is not restricted by the finite volumes of the ions. Namely, the calculated density profile of the multivalent ions is subject to the assumption that the ions are dimensionless as they are in classical PB theory. This is partially the reason that the predicted orientational ordering of multivalent ions is significant only in the close vicinity of the charged plane (figure 3). For example, if the closest approach of ions to the charged plane were taken into account, the ion density would be defined only for $z>a / 2$ [22](see also figure 1). In the model presented, the finite dimensions of the multivalent ions are considered only in the expression for the energy of a single multivalent ion in the electric field (equation (1)); i.e., we take into account only the internal charge distribution of the single multivalent ion while the excluded volume effect is not considered.

The theoretical results presented in figure 2 can motivate further experimental study of the physical properties of a solution of large multivalent ions in contact with a highly charged plane. Recently, experiments with a solution of large phosphotungstate ions $\left(\mathrm{PW}_{12} \mathrm{O}_{40}^{3-}\right)$ in contact with a highly charged monolayer composed of eicosylamine $\left(\mathrm{C}_{20} \mathrm{H}_{40}-\mathrm{NH}_{2}\right)$ were performed $[4,23]$. The diameter $(a)$ of spheroidal $\mathrm{PW}_{12} \mathrm{O}_{40}^{3-}$ ions (see also figure 1) is around $1 \mathrm{~nm}[2,3]$, so the range of values of the parameter $l<1 \mathrm{~nm}$ given in figure 3 seems to be a reasonable selection. In the above-mentioned experiments of Cuvillier et al the $\mathrm{pH}$ value of the solution of phosphotungstate ions was very low [4, 23], so the amine group of eicosylamine facing the solution was fully ionized. Thus the head-group plane of the eicosylamine monolayer was highly positive. The lateral pressure, the area per eicosylamine molecule, and therefore the surface charge density $\sigma$ were varied by a compression barrier. In this way, the surface charge density $\sigma$ of the eicosylamine monolayer was varied between $0.1 \mathrm{As} \mathrm{m}^{-2}$ and approximately $0.8 \mathrm{As} \mathrm{m}^{-2}$ [4] which is also the range of values of $\sigma$ given in figure 3 .

In the present work, we adopted many simplifications (in addition to those already mentioned above) such as, for example, that we did not consider the adsorption of multivalent ions [23, 24]. Also we did not take into account the internal space charge distribution of a single multivalent ion in Gauss's law (see also [5]) and we neglected the possible steric restriction of the orientation of multivalent ions near the charged surface (if the ions are not spherical).

Our present approach is also based on the mean field PB theory, entirely neglecting interionic correlations which become important in highly charged systems and can lead to attractive interactions between equally charged surfaces [25-30]. Yet it takes into account intra-ionic correlations through the constraint that effective charges within a single multivalent ion are separated from each other by a fixed distance $l$ (figure 1).

Nevertheless, using the simple three-state model for the orientational ordering of large multivalent ions and applying the methods of statistical physics, we predicted a non-zero average orientation of multivalent ions in the direction of the electric field, i.e. in the direction of the normal to the charged plate. At small values of $l$ and/or high temperatures and/or low surface charge densities of the charged plate $(\sigma)$, the effect of the preferred orientation of the multivalent ions near the charged plate is negligible. 
In conclusion, we showed that at finite temperatures and high enough surface charge densities $\sigma$, the internal charge distribution within a single multivalent ion (with the internal charges located at large enough distances) may lead to the orientational ordering of these multivalent ions near a charged surface in contact with the solution of multivalent ions.

\section{References}

[1] McLaughlin S 1989 Ann. Rev. Biophys. Biophys. Chem. 18113

[2] Spirlet M R and Busing W R 1987 Acta Cryst. 154137

[3] Lin M M 2001 Appl. Catal. A 2071

[4] Cuvillier N and Rondelez F 1998 Thin Solid Films 327-329 19

[5] Jackson J C 1999 Classical Electrodynamics (New York: Wiley) pp 248-58

[6] Freise V 1952 Z. Elektrochem. 56822

[7] Eigen M and Wicke E 1954 J. Phys. Chem. 58702

[8] Kralj-Iglič V and Iglič A 1996 J. Physique II 6477

[9] Borukhov I, Andelman D and Orland H 1997 Phys. Rev. Lett. 79435

[10] Manciu M and Ruckenstein E 2002 Lamgmuir 185178

[11] Trizac E and Raimbault J L 1999 Phys. Rev. E 606530

[12] Barbero G, Evangelista L R and Olivero D 2000 J. Appl. Phys. 872646

[13] Lue L, Zoeller N and Blankschtein D 1999 Langmuir 153726

[14] Outhwaite C W and Lamperski S 2001 Condens. Matter Phys. 4739

[15] Carnie S L and Torrie G M 1984 Adv. Chem. Phys. 56141

[16] Bhuiyan L B, Outhwaite C W and Bratko D 1992 Chem. Phys. Lett. 193203

[17] Bratko D 1990 Chem. Phys. Lett. 169555

[18] Gonzales-Tovar E, Lozada-Cassou M and Henderson D 1985 J. Chem. Phys. 83361

[19] Borukhov I, Andelman D and Orland H 2000 Electrochim. Acta 46221

[20] Engström S and Wennerström H 1978 J. Chem. Phys. 822711

[21] Bohinc K, Iglič A and May S 2004 Europhys. Lett. 68494

[22] Bohinc K, Kralj-Iglič V and Iglič A 2001 Electrochim. Acta 463033

[23] Cuvillier N, Bonnier M, Rondelez, Paranjape D, Sastry M and Ganguly P 1997 Prog. Colloid Polym. 105118

[24] Borukhov I, Andelman D and Orland H 1999 J. Phys. Chem. 1035042

[25] Kirkwood J G and Shumaker J B 1952 Proc. Natl Acad. Sci. USA 38863

[26] Oosawa F 1968 Biopolymers 61633

[27] Guldbrand L, Jönsson, Wennerström H and Linse P 1984 J. Chem. Phys. 802221

[28] Valleau J P, Ivkov R and Torrie G M 1991 J. Chem. Phys. 95520

[29] Kjellander L, Marčelja S and Quirke J P 1988 J. Colloid Int. Sci. 126194

[30] Lee K C, Borukhov I, Gelbart W M, Liu A J and Stevens M J 2004 Phys. Rev. Lett. 93128101 